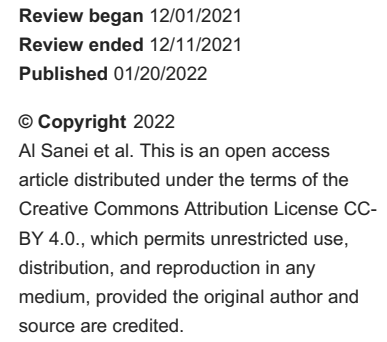

\section{Fetal Brain Injury in Rhesus Isoimmunization Fetus}

\author{
Eman Al Sanei ${ }^{1}$, Mostafa Elbatreek ${ }^{2}$, Rula B. Sallout ${ }^{3}$, Badi Al Baqawi ${ }^{1}$, Bahauddin I. Sallout ${ }^{1}$ \\ 1. Maternal Fetal Medicine, Women's Specialized Hospital, King Fahad Medical City, Riyadh, SAU 2. Neonatal Intensive \\ Care Unit, Children's Specialized Hospital, King Fahad Medical City, Riyadh, SAU 3. Collage of Medicine, Al Faisal \\ University, Riyadh, SAU
}

Corresponding author: Bahauddin I. Sallout, bsallout@kfmc.med.sa

\begin{abstract}
This report presents a rare fetal and neonatal complication brain injury (encephalomalacia and ventriculomegaly) as a consequence of severe fetal anemia resulting from Rhesus ( $\mathrm{Rh}$ ) isoimmunization. A 28 -year-old gravida 4 para 3 woman was referred at $21+4$ weeks of gestation to the fetal medicine clinic as a case of Rh isoimmunization. Fetal ultrasound showed a normal anatomy scan with normal brain structure, but with severe fetal anemia. The patient was treated with multiple intrauterine transfusions, but still developed complications post-transfusions.
\end{abstract}

This case shows that severe cerebral developmental anomalies can occur because of severe fetal anemia secondary to Rh isoimmunization, such as in this case - ventriculomegaly and encephalomalacia. It has been concluded that proper antenatal counseling and early intervention for severe fetal anemia are beneficial to prevent such complications from occurring. It is crucial to consider appropriate antenatal and postnatal radiological imaging for such cases.

Categories: Obstetrics/Gynecology

Keywords: hdrops, intrauterine transfusion, fetal anemia, encephalomalacia, ventriculomegaly

\section{Introduction}

ABO type, Rhesus ( $R h\{D\})$ status, and antibody screening should be determined in all pregnant women at the initial perinatal visit. The most common antigen causing isoimmunization is $R h(D)$. Only $17 \%$ of $R h(D)$ negative women who do not receive prophylaxis immunoglobulin become immunized. Up to 90\% cases of Rh isoimmunization can be prevented by routine postpartum immunoglobulin administration to $\mathrm{Rh}(\mathrm{D})$ negative women, and >99\% cases can be prevented if the immunoglobulin is given during the third trimester and postpartum.

\section{Case Presentation}

A 28-year-old gravida 4 para 3 woman with two living children presented with a medical history of rheumatic heart disease. The patient underwent aortic valve replacement with a tissue valve in 2012, during which she received many units of blood transfusion and developed Rh isoimmunization (blood group: A, Rhnegative and anti-D positive). Her first pregnancy was complicated by hydrops due to which she did not receive an intrauterine blood transfusion (IUT) and was delivered via cesarean section (C/S) prematurely. The second pregnancy was complicated by intrauterine fetal death and terminated before 24 weeks of gestation. The patient's last pregnancy was managed with frequent IUTs, and she delivered a healthy newborn via cesarean section $(\mathrm{C} / \mathrm{S})$ at 36 weeks of gestation.

During the current pregnancy, the patient presented for the first time at 21 weeks and three days of gestation with an uneventful pregnancy. The anatomy scan revealed normal fetal anatomy without signs of hydrops but with middle cerebral artery-peak systolic velocity (MCA-PSV) at $42.8 \mathrm{~cm} / \mathrm{s}$ (1.6 multiple of median $\{$ MoM\}; Figure 1). The patient underwent fetal blood sampling and IUT of $15 \mathrm{~mL}$, and weekly assessment of MCA-PSV was accordingly done with multiple IUTs as illustrated in Table 1 . At 26 weeks of gestation, after four episodes of IUT, the brain ultrasound showed bilateral severe ventriculomegaly, dilated third ventricle, and cerebellar cyst, likely representing periventricular leukomalacia due to brain hypoxia (Figure 2). The patient was counseled on the poor outcome of this rare fetal anemia complication and repeated IUT. The pregnancy continued until 34 weeks and five days, and the patient delivered a baby girl via an emergency $\mathrm{C} / \mathrm{S}$. 


\section{Cureus}

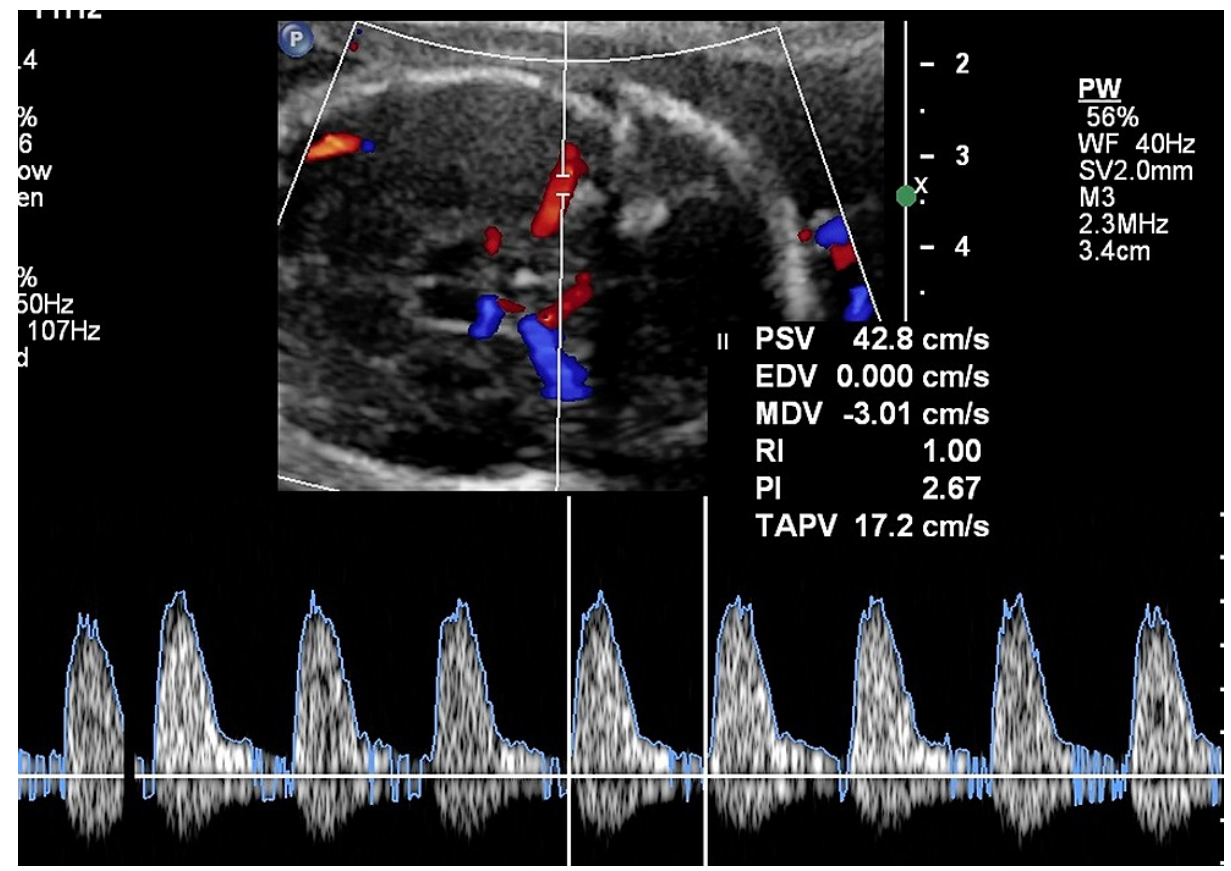

FIGURE 1: Doppler showing peak systolic velocity of 29.4 (1.60 MoM)

MoM: multiple of median

\begin{tabular}{|c|c|c|c|c|}
\hline Transfusion cycle no. & Gestational age & MCA-PSV (cm/s) & MCA-PSV (MoM) & Blood volume transfused $(\mathrm{mL})$ \\
\hline 1 & 21 weeks and 5 days & 42.8 & 1.6 & 15 \\
\hline 2 & 24 weeks and 2 days & 52.8 & 1.7 & 58 \\
\hline 3 & 25 weeks and 4 days & 62.5 & 1.9 & 44 \\
\hline
\end{tabular}

TABLE 1: Fetal MCA-PSV in relation to each intrauterine blood transfusion cycle

MCA-PSV: middle cerebral artery-peak systolic velocity; MoM: multiple of median 


\section{Cureus}

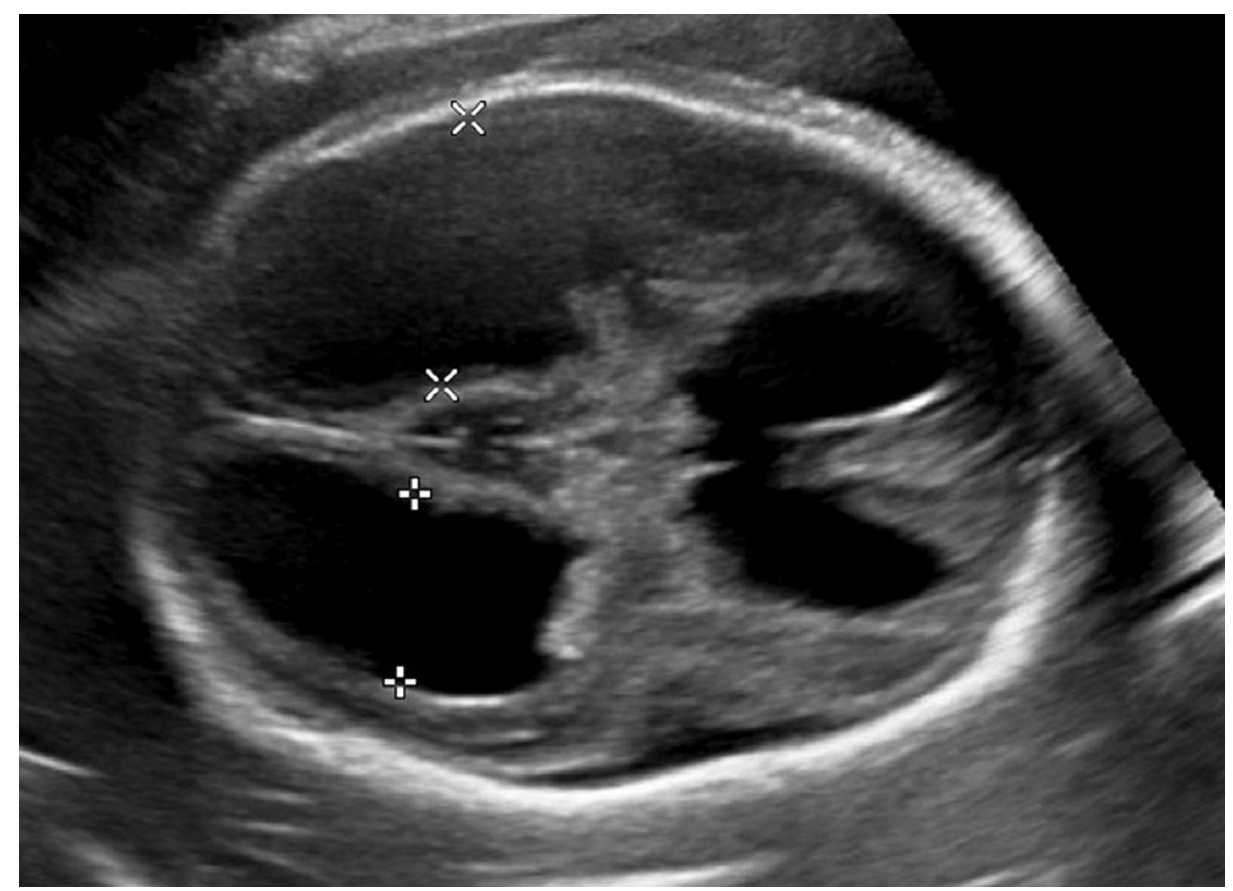

\section{FIGURE 2: Ultrasound after the fourth transfusion showing severe ventriculomegaly $(24 \mathrm{~mm})$}

Newborn findings are shown in Table 2. The newborn was admitted to the neonatal intensive care unit due to respiratory distress, anemia, and neonatal jaundice and was placed on continuous positive airway pressure. The newborn received O-negative blood transfusion at birth. Exchange transfusion and immunoglobulin were given 2.5 hours after birth. The newborn was started on intensive phototherapy. The serum bilirubin level decreased gradually to below the phototherapy zone after 40 hours, and the newborn was weaned gradually from oxygen to room air over a period of two days. The newborn had difficulty tolerating oral feeding, taking approximately two weeks to shift from tube to oral feeding.

\begin{tabular}{|l|l|}
\hline Newborn findings & Values \\
\hline APGAR score & 7 and 8 at 1 and 10 min, respectively \\
Cord pH & Venous, 7.23 and arterial, 7.14 \\
Weight & $2080 \mathrm{~g}$ (falling on the 10 th and 50 th percentile) \\
\hline Length & $41 \mathrm{~cm}$ (third percentile) \\
Head circumference & $32.5 \mathrm{~cm}$ (50th percentile) \\
Bilirubin & $149 \mathrm{mmol} / \mathrm{dL}$ (jaundiced) \\
Hemoglobin & $4.6 \mathrm{~g} / \mathrm{dL}$ \\
\hline Physical examination & Irritable, with high-pitched cry. Hypertonicity of the upper limbs. Spasticity of the lower limbs \\
\hline
\end{tabular}

\section{TABLE 2: Newborn findings}

APGAR: appearance, pulse, grimace, activity, and respiration

Magnetic resonance imaging of the brain showed bilateral marked lateral ventricular dilatation, which appeared to be passive due to parenchymal volume loss with a very thin cortical mantel. The third and fourth ventricles were not dilated. No obstructive lesions were identified at the level of the foramen of Monro. Cystic encephalomalacia changes in the cerebellum were noticed (Figure 3). The newborn was discharged home with a follow-up appointment at the physiotherapy, neurology, and high-risk neonatal clinic in addition to follow-up at the clinic four weeks post discharge. The newborn showed bilateral optic nerve 


\section{Cureus}

atrophy, hyperreflexia, and contracture on the lower limbs that needed surgical fixation; frequent choking on feeds; and findings of global developmental delay.

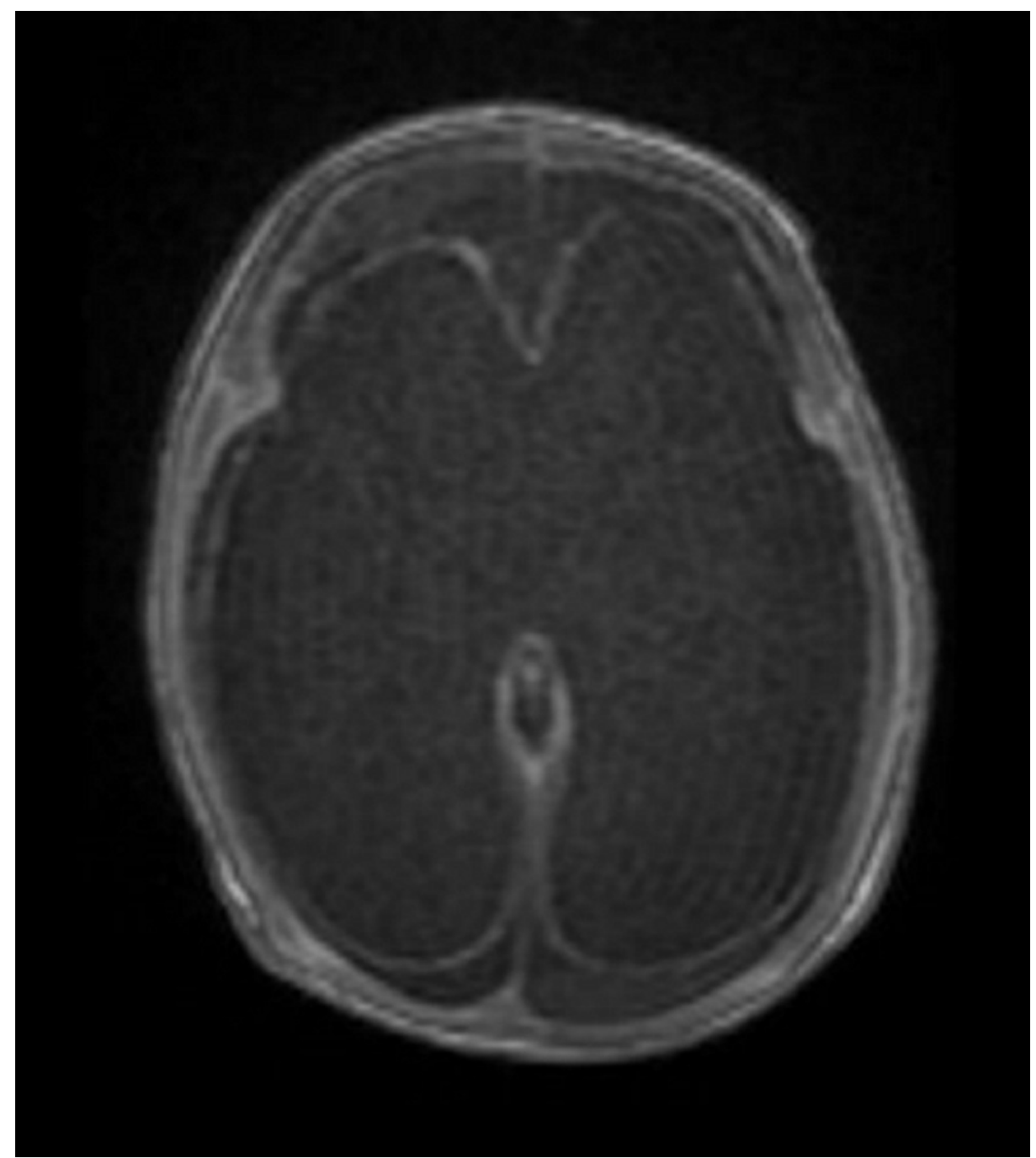

FIGURE 3: Postnatal brain magnetic resonance imaging of a patient with severe brain volume loss and mantel index thinning

\section{Discussion}

IUT is the gold standard of fetal anemia treatment, with a good prognosis and minor risk of fetal loss (1-3\%) in uncomplicated procedures. However, the risk increases to about $20 \%$ in hydropic fetuses [1]. Fetal brain lesions can occur as complications of severe and prolonged intrauterine fetal anemia, leading to brain anoxia mainly due to delayed referral for intrauterine transfusion or technical difficulty. Dildy et al. reported a case of a porencephalic cyst related to a difficult intrauterine transfusion [2]. Brain lesions that are seen after IUT in severely anemic fetuses mimic sonographic neurological appearance similar to that observed in monochorionic twins after the demise of one, which supports the hypothesis that brain anomalies can develop after severe fetal anemia [3]. In addition, unilateral cerebellar hypoplasia was related to intrauterine ischemic or vascular injuries [4]. However, the patient in the current case developed encephalomalacia as a rare complication of fetal anemia due to $\mathrm{Rh}$ isoimmunization.

In a retrospective study, Pasman et al. have reported 135 IUTs in 56 fetuses, with minimal adverse effects of approximately $10 \%$. They found that severe fetal effects occurred in only $1.5 \%$, and hydrops and IUT in the free lope were a significant predisposing factor. However, IUT in the late gestation was associated with good fetal and neonatal outcomes [5].

The risk of anemia is considered high when MCA-PSV reaches $\geqslant 1.5 \mathrm{MoM}$, whereas levels below this value indicate that the fetus is not anemic or does not call for interventions [6]. MCA-PSV, which was normalized after transfusion, has been considered a standard for the detection of fetal anemia and the timing of IUT [7]. In few cases, it has been reported that IUT in the early second trimester was associated with higher fetal loss 
(6.7\%) and increased probability of prenatal intracranial hemorrhage [8]. Despite successful IUT, fetuses with severe anemia were at increased risk of cerebral damage mainly between 24 and 32 weeks of gestation, in which the proliferation of neuroblast and differentiation of cerebral cortex take place [9].

The LOTUS study found that children (two to 17 years) who received IUT had normal neurodevelopment. This shows the safety of IUTs regardless of the number of procedures, with rare complications mainly related to premature delivery, hydrops fetuses, and low hemoglobin rather than the number of transfusions or procedures itself [10]. Severe anemia due to red blood cell alloimmunization seems to increase the risk of cerebellar damage when the fetal hemoglobin reaches a critical value $(<2 \mathrm{~g} / \mathrm{dL})$. Moreover, hypoxic-ischemic encephalopathy takes seven to 14 days to be diagnosed by radiological modality [11].

\section{Conclusions}

IUT is the gold standard for fetal anemia treatment. In cases of Rh isoimmunization, delayed treatment or complicated IUT procedures can cause fetal brain anoxia and brain lesions. Thus, timely and careful intervention by an experienced maternal fetal medicine specialist is crucial for superior fetal and neonatal outcomes.

\section{Additional Information \\ Disclosures}

Human subjects: Consent was obtained or waived by all participants in this study. King Fahad Medical City Institutional Review Board issued approval NA. Conflicts of interest: In compliance with the ICMJE uniform disclosure form, all authors declare the following: Payment/services info: All authors have declared that no financial support was received from any organization for the submitted work. Financial relationships: All authors have declared that they have no financial relationships at present or within the previous three years with any organizations that might have an interest in the submitted work. Other relationships: All authors have declared that there are no other relationships or activities that could appear to have influenced the submitted work.

\section{References}

1. Grab D, Paulus WE, Bommer A, Buck G, Terinde R: Treatment of fetal erythroblastosis by intravascular transfusions: outcome at 6 years. Obstet Gynecol. 1999, 93:165-8. 10.1016/s0029-7844(98)00421-9

2. Dildy GA 3rd, Smith LG Jr, Moise KJ Jr, Cano LE, Hesketh DE: Porencephalic cyst: a complication of fetal intravascular transfusion. Am J Obstet Gynecol. 1991, 165:76-8. 10.1016/0002-9378(91)90227-i

3. Carbonne B, Nguyen A, Cynober E, Castaigne V, Cortey A, Brossard Y: Prenatal diagnosis of anoxic cerebral lesions caused by profound fetal anemia secondary to maternal red blood cell alloimmunization. Obstet Gynecol. 2008, 112:442-4. 10.1097/AOG.0b013e3181656a11

4. Benbir G, Kara S, Yalcinkaya BC, Karhkaya G, Tuysuz B, Kocer N, Yalcinkaya C: Unilateral cerebellar hypoplasia with different clinical features. Cerebellum. 2011, 10:49-60. 10.1007/s12311-010-0225-2

5. Pasman SA, Claes L, Lewi L, et al.: Intrauterine transfusion for fetal anemia due to red blood cell alloimmunization: 14 years experience in Leuven. Facts Views Vis Obgyn. 2015, 7:129-36.

6. Mari G, Deter RL, Carpenter RL, et al.: Noninvasive diagnosis by Doppler ultrasonography of fetal anemia due to maternal red-cell alloimmunization. Collaborative Group for Doppler Assessment of the Blood Velocity in Anemic Fetuses. N Engl J Med. 2000, 342:9-14. 10.1056/NEJM200001063420102

7. Yalinkaya A, Evsen MS, Celik Y, Sak ME, Soydinc HE, Taner MZ: Intrauterine blood transfusion in immune hydrops fetalis, corrects middle cerebral artery Doppler velocimetry very quickly. Bosn J Basic Med Sci. 2012, 12:37-40. 10.17305/bjbms.2012.2532

8. Yinon Y, Visser J, Kelly EN, Windrim R, Amsalem H, Seaward PG, Ryan G: Early intrauterine transfusion in severe red blood cell alloimmunization. Ultrasound Obstet Gynecol. 2010, 36:601-6. 10.1002/uog.7696

9. Lee C, Kim DW, Jeon GS, Roh EJ, Seo JH, Wang KC, Cho SS: Cerebellar alterations induced by chronic hypoxia: an immunohistochemical study using a chick embryonic model. Brain Res. 2001, 901:271-6. 10.1016/s0006-8993(01)02362-9

10. Verduin EP, Lindenburg IT, Smits-Wintjens VE, et al.: Long-term follow up after intra-uterine transfusions; the LOTUS study. BMC Pregnancy Childbirth. 2010, 10:10.1186/1471-2393-10-77

11. Ghi T, Brondelli L, Simonazzi G, et al.: Sonographic demonstration of brain injury in fetuses with severe red blood cell alloimmunization undergoing intrauterine transfusions. Ultrasound Obstet Gynecol. 2004, 23:428-31. 10.1002/uog. 1035 